\title{
Synthetic studies on gibberellins and antheridiogens
}

\author{
Lewis N. Mander \\ Research School of Chemistry, Australian National University, Canberra, 2601 Australia.
}

\begin{abstract}
Many of the biologically more interesting gibberellins can only be obtained from natural sources in very small amounts. An extensive range of new methodology has therefore been developed for the synthesis of these compounds and a number of structurally related growth factors from fern gametophytes.
\end{abstract}

\section{INTRODUCTION}

The great majority of the 70 odd known gibberellins ("GAs") have been isolated in only very small quantities. In order to confirm provisional structure assignments and to obtain sufficient material for thorough biological investigations it has therefore been necessary to synthesise many of the new compounds from the more readily available fungal $\mathrm{GAs}$. gibberellic acid $\left(\mathrm{GA}_{3}\right) \mathbf{1}, \mathrm{GA}_{7} 2, \mathrm{GA}_{4} \mathbf{3}$. and $\mathrm{GA}_{13} \mathbf{5}$. While surveying the field in 1984. however, it became apparent that there were no procedures available which would provide access to the 20-oxo-GAs, especially $\mathrm{GA}_{19} 6$ which plays such an important role in biosynthetic pathways involving early 13-hydroxylation. or to GAs which were functionalised in the C-ring (ref. 1). This latter group is currently represented by three compounds hydroxylated at $\mathrm{C}(11)$ and eleven at $\mathrm{C}(12)$, including $\mathrm{GA}_{32} 7$, the biologically most potent of all GAs discovered to date. In addition to the known derivatives, however, tentative structural assignments for recently isolated compounds are consistent with hydroxylation at $\mathrm{C}(11)$ or $\mathrm{C}(12)$ also. while others appear to have double bonds in the C-ring. We have therefore embarked upon a synthetic program aimed at providing access to these GAs. At the same time we have become involved in the study of a new group of naturally occurring gibberellin-like growth substances termed antheridiogens which promote antheridia formation in fern gametophytes from the family Schizaeaceae (ref. 2) e.g. our recently completed conversion of $\mathrm{GA}_{7} 2$ into antheridic acid 8, the principal antheridiogen from Anemia phyllitidis (ref. 3). The very tiny quantities of available materials in this field have made structure assignments very difficult. however, and it is has been necessary to combine synthesis with spectroscopic studies in order to determine the constitution of new compounds. This effort has very recently led to the elucidation of structure for the respective antheridiogens from Anemia mexicana and Lygodium japonicum. and provided adequate supplies of these materials for the first time.

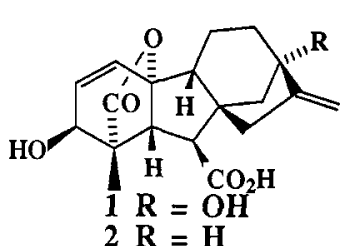

$2 \mathrm{R}=\mathrm{H}$

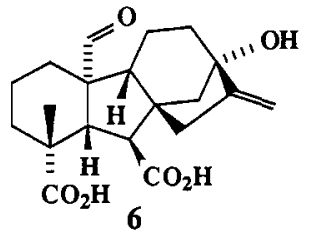

\section{CONVERSIONS OF $\mathrm{C}_{19}$ GIBBERELLINS INTO $\mathrm{C}_{20}$ ANALOGUES}

Access to many $C_{20}$ GAs which lack a hydroxyl at $\mathrm{C}(13)$ is feasible from $\mathrm{GA}_{13} 5$ and several syntheses have been reported (ref. 1). In contemplating the preparation of $\mathrm{GA}_{19} 6$, however. there is a choice between dismantling and then reconstructing the D-ring to allow introduction of the 13-hydroxyl, or of utilising $\mathrm{GA}_{3} 1$ as a substrate. Both options pose major challenges. We chose the latter course. even though this required manipulation of a notoriously labile system and the introduction of the twentieth carbon atom into the sterically most hindered part of the molecule. In view of this steric hindrance, we elected to use an intramolecular approach based on the cyclopropanation of a $\Delta 1(10)$ olefinic bond by a diazoacetyl function located at $C(19)$ and then to delete the surplus $C(1)-C(20)$ bond by reduction in liquid ammonia with lithium 
Scheme 1

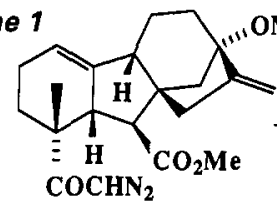
OMOM<smiles>CC(C)(C)C1CCCCC1</smiles>

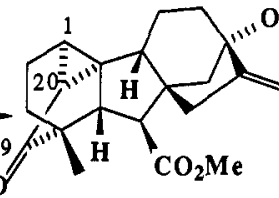

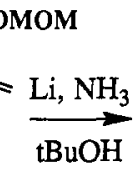

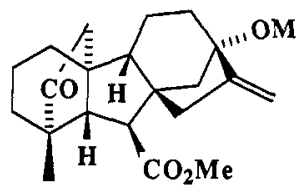
$\underset{\text { 2. deprotection }}{\stackrel{\mathrm{KH}, \mathrm{DMF}, \mathrm{O}_{2}}{\longrightarrow}} 6$

metal. The prospect of subjecting such complex molecules to the vigorous conditions of the Birch reduction was somewhat daunting, but in practise the reactions proceed smoothly and efficiently; it was even possible to avoid reduction of the B-ring methoxycarbonyl group. The successful outcome of these considerations is outlined in Scheme 1 (ref. 4); it should be noted that the Birch reduction process was also utilised to dismantle the A-ring functionality in the preparation of the required olefinic acid precursor. The last stage of the conversion required the oxidative fission of the $\mathrm{C}(19)-\mathrm{C}(20)$ bond and although steric crowding rendered most procedures ineffective, it was eventually possible to achieve this simply by oxygenation of the potassium enolate which led directly to the desired carboxy aldehyde.

\section{SYNTHESIS OF 12-HYDROXY GIBBERELLINS}

There appeared to be two basic options for the insertion of functional groups into the C-ring of GAs: to extend the A-ring functionality, or to effect a transannular process from substituents attached to the D-ring. We have successfully pursued the former approach to introduce an olefinic bond into the $\Delta 9(11)$ position (vide infra). but in view of the need to re-establish the correct $9 \beta$ stereochemistry we chose first to concentrate on the transannular strategy. The outcome of these efforts has been the process outlined in Scheme 2 whereby

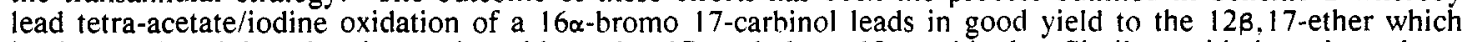
is then reduced by zinc in acetic acid to the 17-methylene 126-carbinol. Similar oxidations have been reported in the related kaurenoid group of compounds, but in these cases the reactions proceed via a chair conformation of the $\mathrm{C}$-ring to effect functionalisation at $\mathrm{C}(\mathrm{I} 1)$ even though the ground state is normally boatlike. In the gibberellin series, however, it appears that the boat conformation of the $C$-ring lies in a deeper

Scheme 2

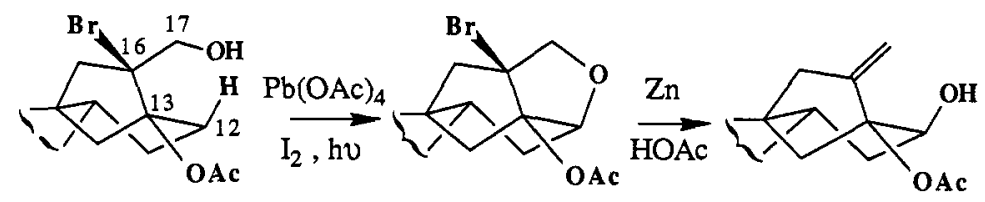

energy well and that the transition state is therefore based on this geometry. The methodology is compatihle with a wide range of functionality elsewhere in the substrate. including the labile $\triangle(1)-3 \beta$-ol array of GA3 1 (protected simply as the acetate) which we hoped to preserve in our attempts to make $\mathrm{GA}_{32} 7$. Thus, it has been possible to prepare $\mathrm{GA}_{69} 9 . \mathrm{GA}_{70} 10, \mathrm{GA}_{58} 11, \mathrm{GA}_{71} 12$, and $\mathrm{GA}_{30} 13$ (ref. 5). For reasons which were not altogether apparent, the procedures worked best when a 13-acetoxy function was present and it was therefore more effective to begin with $\mathrm{GA}_{3} 1$ even though it was then necessary for most objectives to remove the 13-oxy function at a later stage. This was carried out most efficiently by samarium iodide reduction of a 13-acetoxy 12-oxo- derivative (although this is clearly not compatible with a $\Delta^{\prime}$-olefinic bond in the A-ring).
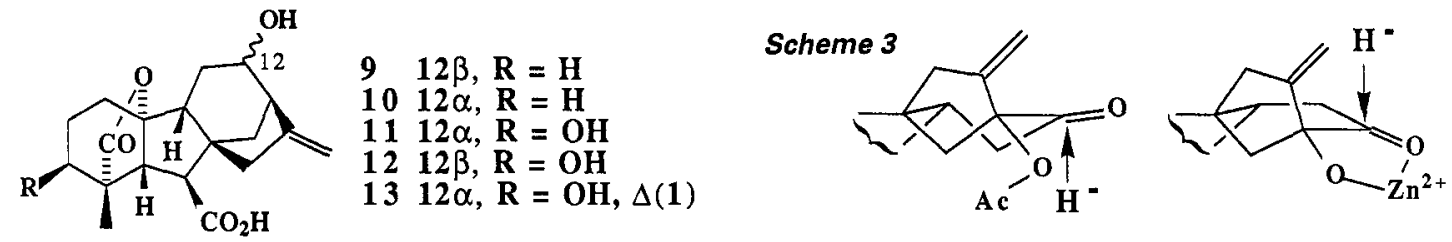

which also re-establishes the $12 \beta$-stereochemistry. To gain access to the $12 \alpha$-stereochemistry, however. is more difficult. $S S_{N} 2$-Substitution processes fail. while simple hydride reduction of the 12-one function returns the 12-B stereochemistry. Our preferred method has been the reduction of the 13-hydroxy-12-oxo system in dimethyl formamide with zinc borohydride. This gave a $3: 2$ mixture of $12 \alpha .13-$ and $12 \beta .13$ diols. presumably as a consequence of chelation which flattens the C-ring. allowing better access by the hydride reagent to the upper $\pi$-face of the $C(12)$ carbonyl group (Scheme 3). Removal of the unwanted 13-hydroxyl from the $12 \alpha$ products was achieved by stannane reduction of the 13-methyl oxalates (ref. 6) (after protection of the 12hydroxyl by acetylation). The remaining challenge in this area of GA synthesis was the preparation of $\mathrm{GA}_{32}$ 7 and this was achieved as outlined in Scheme 4 following yet another method developed by the MacMillan group (ref. 7 ).
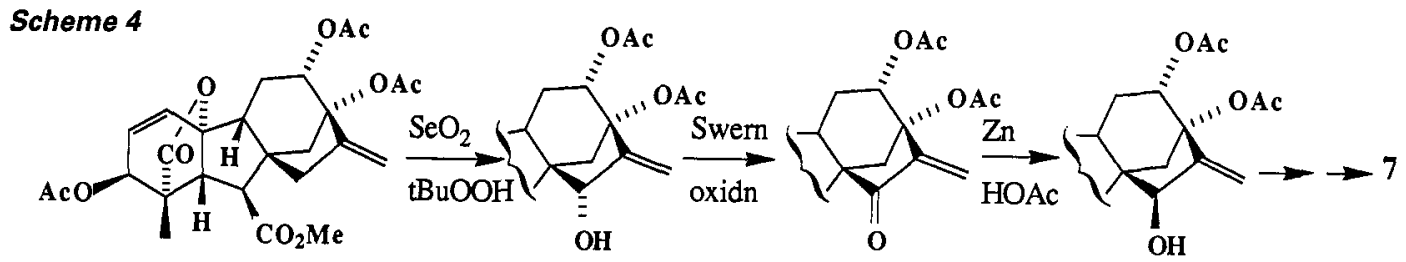
STRUCTURE AND SYNTHESIS OF THE ANEMIA MEXICANA ANTHERIDIOGEN

Scheme 5

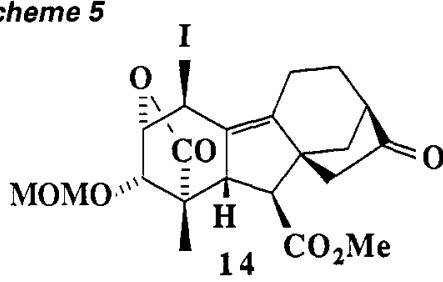

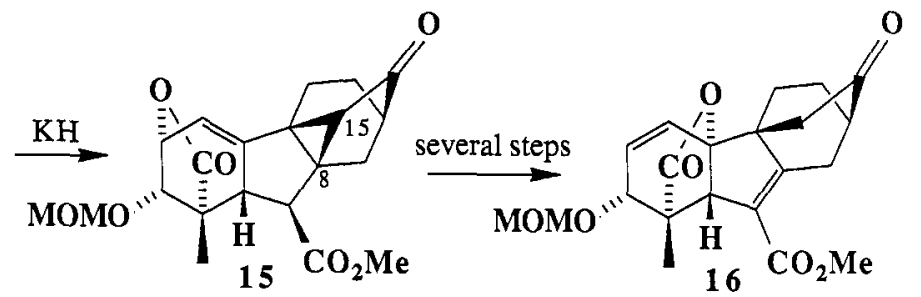

The key sequence in our recent synthesis of antheridic acid 8 from $\mathrm{GA}_{7} 2$ had been the intramolecular alkylation conducted on iodide 14 to form cyclopropyl ketone 15 followed by cleavage of the $C(8)$. $C(15)$ bond to afford 16 (initiated by enolisation of the B-ring ester function) (Scheme 5). Just as this study was drawing to a close our attention was drawn to a speculation that a new antheridiogen from Ancmia mexicana (ref. 8) might have the cyclogibberellin structure 17. We therefore prepared the $3 \beta$-epimer of 15 . i.e. 19. by a simple adaptation of the earlier methodology, and re-established the

$\Delta-19.10$ lactone functionality by treatment with diphenylboron bromide. thereby obtaining 20 (Scheme 6). After spectroscopic comparisons between the synthetic intermediates and the natural substance we concluded that the structure hypothesis was broadly correct, but that the antheridiogen should be reformulated as $\mathbf{1 8}$. The intermediate 20 was therefore converted to mesylate 21 and this was treated with lithium acetate in

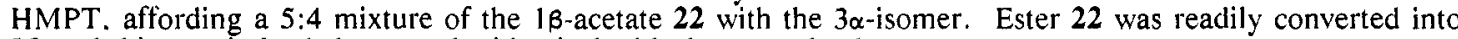
18 and this was indeed shown to be identical with the natural substance.

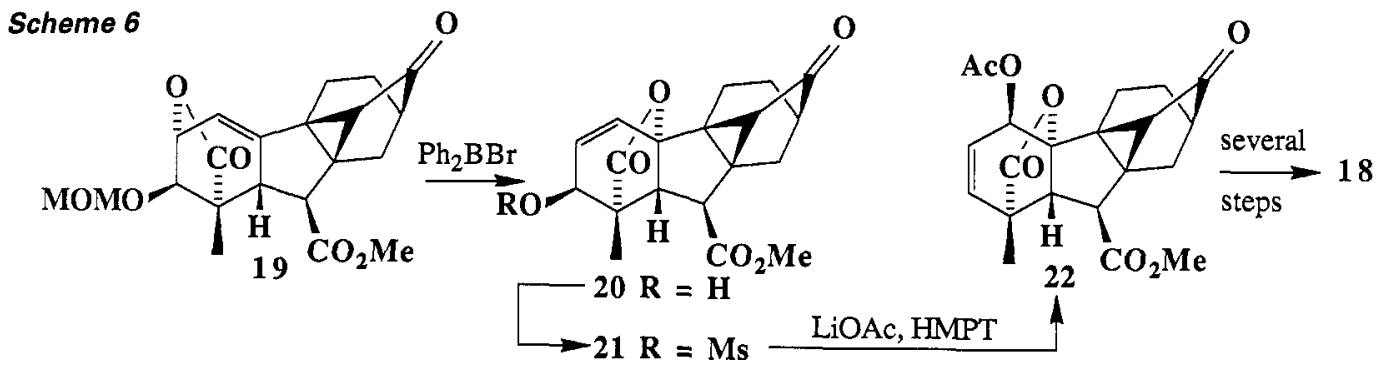

\section{SYNTHESIS OF THE MORE POTENT ANTHERIDIOGEN FROM \\ L. JAPONICUM}

The methyl ester of $\mathrm{GA}_{9} 4$ was shown some years ago to be an antheridiogen in the fern Lygodium japonicum (ref. 9). The co-occurrence of a more potent substance was suspected for some time and was eventually isolated in nanogram quantities (ref. 10). Mass spectrometry revealed that it was similar to 4 but that it possessed an additional double bond or ring. After comparison with a range of possible candidates prepared by synthesis. it became apparent that the most likely structure was 26 . We therefore drew once again on the experience gained during our synthesis of antheridic acid $\mathbf{8}$. Gibberellenic acid monomethyl ester $\mathbf{2 3}$ had been used as a substrate in some of the early studies. We reasoned that it should be possible to convert this compound into 24 and then effect an iodolactonisation to give 25 . Base promoted elimination of the $9 \beta$-iodo group followed by reintroduction of the 17-methylene group was then expected to afford 26 as outlined in Scheme 7. This proposal was successfully realised in practise and 26 was shown to be identical with the natural antheridiogen.

\section{Scheme 7}

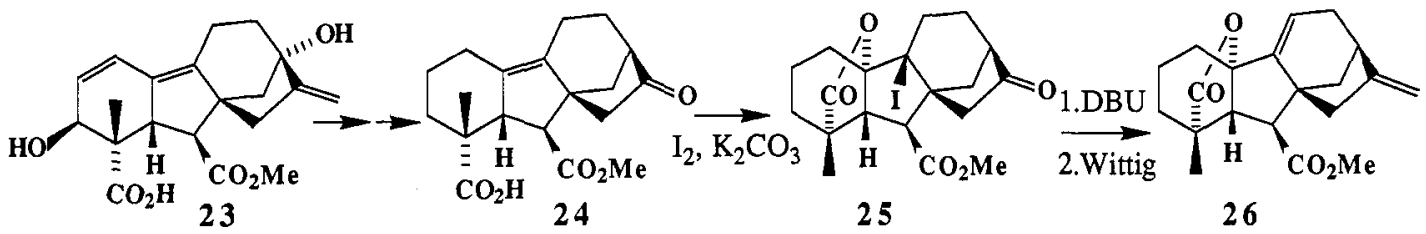




\section{SECOND GENERATION SYNTHESIS OF THE ANEMIA AND LYGODIUM ANTHERIDIOGENS}

Although we had now achieved syntheses of all three kinds of known antheridiogens, we were dissatisfied with an early procedure common to all of the preparations, namely the treatment of $\Delta 1$-3-hydroxy-GAs with hydrazine (ref. 11) to afford $\Delta^{1,9}$-diene 19-carboxylic acids, e.g. 23. GA, 1 and $\mathrm{GA}_{7} 2$ react in an acceptable $40 \%$ yield (given that this occurs early in each of the sequences), but as a consequence of partial hydrolysis the amount of useful product is halved for GA esters. the use of which is necessary to allow subsequent discrimination between the two carboxy functions. In an attempt to bypass this step in the synthesis of the Anemia antheridiogens, therefore, we examined the allylic bromination of the more efficiently obtained lactone 27 (c.f. ref. 12) in the hope that the rearranged bromide $\mathbf{2 8}$ would be obtained and that this would serve as an effective precursor to cyclopropyl ketone 20 . In the event, it was not possible to obtain 28 cleanly, but when an excess of reagent was used, dibromide 29 was formed in high yield and this was smoothly converted into 30 and thence 31 . From 31 it was possible not only to prepare both Anemia antheridiogens 8 and 18 [the unwanted 11-bromo group was removed by E2 elimination, followed by hydrogenation at a suitable stage] but also to gain access to the Lygodium antheridiogen $\mathbf{2 6}$ via direct hydrogenolysis (Scheme 8 ).

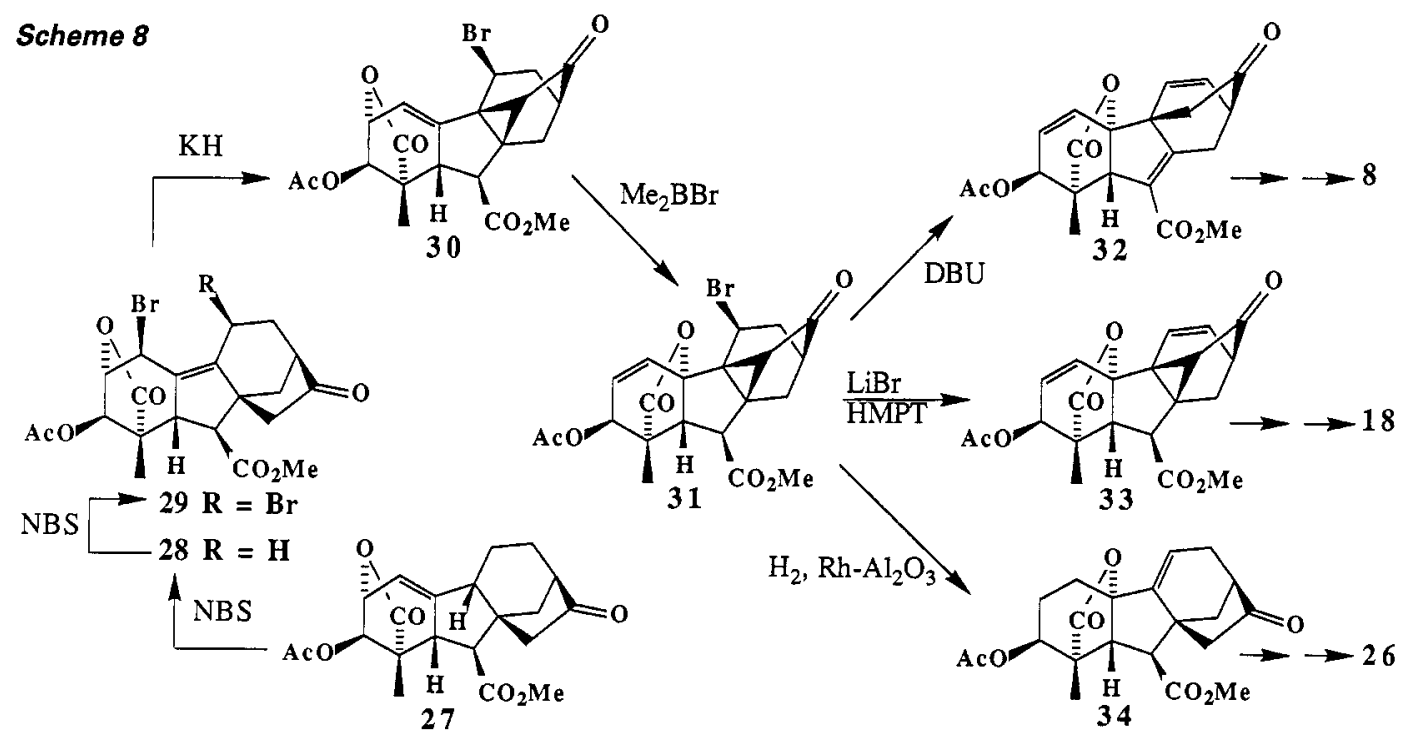

Acknowledgements The author gratefully acknowledges major contributions by many coworkers involved in the studies described above, and in particular expresses his considerable debt to A. Chu. R.D. Dawe, M. Furber, R.P. Pharis, J.V. Tumer, and H. Yamane.

\section{REFERENCES}

1. A. Crozier, ed. "The Biochemistry and Physiology of Gibberellins". Vol. I and II. Praeger. New York. (1983).

2. W. Näf, K. Nakanishi, M. Endo, Bol. Rev. 41, 315-359 (1975).

3. M. Furber and L.N. Mander, J. Am. Chem. Soc. 109. 6389-6396 (1987).

4. R.D. Dawe, L.N. Mander and J.V. Turner, Tetrahedron Lett. 26. 363-366 (1985).

5. A. Chu and L.N. Mander. Tetrahedron Lett. submitted for publication.

6. S.C. Dolan and J. MacMillan, J. Chem. Soc. Chem. Conmun. 1588-1589 (1985).

7. S.C. Dolan, D.W. Holdup, M. Hutchison and J. MacMillan, J. Chem. Soc. Perkin Trans I $651-654$ (1985).

8. J.E. Nester, S. Veysey and R.C. Coolbaugh, Planta 170, 26-33 (1987). Structure 17 was suggested by Professor N. Takahashi and Dr H. Yamane after examination of NMR data provided by Dr J. Nester (personal communication).

9. H. Yamane, N. Takahashi. K. Takeno, M. Furuya. Planta 147. 251-256 (1979).

10. N. Takahashi and $\mathrm{H}$. Yamane, personal communication.

11. J.S. Moffat, J. Chem. Soc. 3045-3049 (1960).

12. P.S. Kirkwood, J. MacMillan and M.L. Sinott, J. Chem. Soc. Perkin Trans / 2117-2121 (1980). 